\title{
Noch so ein Sieg, und wir sind verloren. Der Nationalstaat nach der Finanzkrise
}

\author{
Wolfgang Streeck
}

Zusammenfassung: Hoffnungen auf eine Rückkehr des nationalen Interventionsstaats im Gefolge der Finanzkrise erscheinen verfrüht. Die zur Krisenbekämpfung ergriffenen Maßnahmen beschleunigen einen seit Jahrzehnten anhaltenden Trend zu immer höherer Staatsverschuldung. Wie in anderen Nationalstaaten auch, so wird der für diskretionäre Politik verfügbare Anteil des Bundeshaushalts in den nächsten Jahren gegen Null tendieren. Das Problem einer möglichen Erschöpfung politischer Gestaltungsmöglichkeiten wird durch die von der Großen Koalition in das Grundgesetz aufgenommene „Schuldenbremse“ weiter verschärft, auch wenn mit einer Einhaltung der Vorschriften zur Beseitigung des strukturellen Defizits nicht wirklich zu rechnen ist.

Schlüsselwörter: Finanzkrise $\cdot$ Fiskalkrise $\cdot$ Staatsinterventionismus $\cdot$ Regierbarkeit

\section{Another such victory and we are ruined: the financial crisis and national state capacity}

\begin{abstract}
The rumors about an impending resurrection of state interventionism in the wake of the global financial crisis may be greatly exaggerated. The measures taken for crisis management have accelerated a decade-old trend toward ever higher public debt. Like in other nation-states, the share of the German Federal budget that is available for discretionary spending will tend toward zero in coming years. The problem of a possible exhaustion of governing capacity will be exacerbated by the balanced budget amendment to the constitution as agreed by the Grand Coalition in 2009. This holds even though it is highly improbable that the new deficit reduction targets will be met.
\end{abstract}

Keywords: Financial crisis $\cdot$ Fiscal crisis $\cdot$ State interventionism $\cdot$ Governability

MPIfG Journal Article

Wolfgang Streeck: Noch so ein Sieg, und wir sind verloren: Der Nationalstaat nach der Finanzkrise. In: Leviathan $38(2), 159$ - 173 (2010). Nomos

The original publication is available at the publisher's web site: http://dx.doi.org/10.1007/s11578-010-0079-5

The MPIfG Journal Articles series features articles by MPIfG researchers and visiting scholars published in peer-reviewed journals. Max Planck Institute for the Study of Societies (MPIfG) Cologne | www.mpifg.de

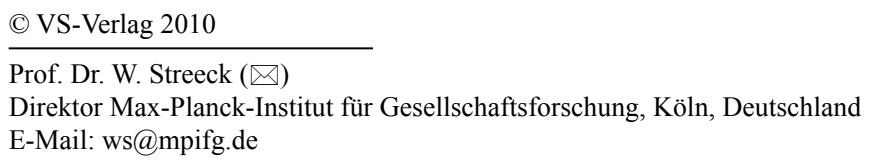


War die Rettung der Banken vor sich selbst, und der Wirtschaft vor den Banken, und der Gesellschaft vor der Wirtschaft die Stunde des Staates: der Rückkehr von Staat und Politik als Souverän über einen Markt, aus dem die Liberalisierung sie fast schon vertrieben hatte? So sind die Ereignisse von 2008 und 2009 zunächst von vielen gesehen worden. Und in der Tat: ohne das Eingreifen der öffentlichen Gewalt - ohne Injektion einer mächtigen Dosis staatlicher Autorität in den Gang der wirtschaftlichen Dinge - stünden wir heute wohl vor den Trümmern des Kapitalismus, so wie wir ihn zu kennen geglaubt haben. Und es waren nicht irgendwelche Nichtregierungsorganisationen oder Internationale Nichtregierungsorganisationen, nicht die zahlreichen Bewohner des Paralleluniversums der ,international governance“ - nicht die Baseler Bank für Internationalen Zahlungsausgleich, nicht der Internationale Währungsfonds, schon gar nicht die Europäische Union - von denen die Rettung kam, sondern die großen Nationalstaaten, allen voran die USA, in Europa Deutschland, Frankreich und Großbritannien, und in Asien der nationalste aller Nationalstaaten, China. Sie allein handelten und waren in der Lage zu handeln, als es ernst wurde.

Haben wir es also hier mit der Wiederkehr eines nationalstaatlich organisierten Kapitalismus zu tun? Zunächst lässt sich feststellen, dass Handlungsfähigkeit nicht auch Entscheidungsfreiheit bedeuten muss. $\mathrm{Zu}$ den staatlichen Rettungsmaßnahmen - dem Aufkauf der falsch bewerteten Wertpapiere und der Auflegung riesiger fiskalischer Stimulus-Programme - gab es nach verbreiteter Ansicht keine Alternativen: Nach erfolgter Geiselnahme der Gesellschaft durch die Finanzbranche konnten die Regierungen nicht anders, als das geforderte Lösegeld zu entrichten (vgl. Ferguson u. Johnson 2009a, b). Tatsächlich schwiegen selbst die fundamentalistischsten Vertreter der reinen Marktlehre, als es darum hätte gehen können, den Staaten ihre Rückkehr in den Markt doch noch auszureden. Und was die deutsche Bundesregierung angeht, so hatte sie gleich in mehrerlei Hinsicht keine Wahl: Hätte sie anfänglichen Versuchungen nachgegeben, zur Verteidigung ihres damaligen Konsolidierungskurses die Neuverschuldung kleinzuhalten, wäre das nicht zuletzt von anderen Staaten mit weniger exportorientierten Volkswirtschaften als Trittbrettfahren aufgefasst worden und hätte zweifellos Vergeltungsmaßnahmen zur Folge gehabt.

Freilich: die Rettung des Kapitalismus verlangte von den Nationalstaaten die Aufnahme einer gigantischen Hypothek, die nun viele Jahre lang wird abgezahlt werden müssen, auch dann noch, wenn, was immerhin nicht auszuschließen ist, der Finanzsektor und, später, die Wirtschaft insgesamt längst saniert sein werden. Im Folgenden soll die Möglichkeit untersucht werden, dass die Rettung der Marktwirtschaft durch den Staat für diesen ein Pyrrhussieg gewesen sein und der unter Einsatz gigantischer öffentlicher Finanzmittel vollbrachte politisch-ökonomische Kraftakt der Jahre nach 2008 eben die staatliche Handlungsfähigkeit, von der doch offenbar für die Stabilität von Wirtschaft und Gesellschaft so viel abhängt, auf absehbare Zeit ruiniert haben könnte. Besonders beunruhigend erscheint dabei, dass die (national-) staatlichen Rettungsmaßnahmen einen seit langem wirksamen Trend in den öffentlichen Finanzen demokratischer Interventionsstaaten verstärkt und beschleunigt zu haben scheinen, der auf eine zunehmende Erschöpfung fiskalischer politischer Reaktionsmöglichkeiten hinausläuft - und damit auf eine säkulare Erosion der politischen Kapazität zu distributivem und investivem Handeln. Hier denken wir an die in den meisten Staaten anscheinend chronische Tendenz zu einer Unterdeckung 
der öffentlichen Haushalte, die nun schon über Jahrzehnte hinweg eine wachsende Staatsverschuldung verursacht hat, die von einem ständigen Zuwachs des Anteils der feststehenden zu Lasten der politisch disponiblen Staatsausgaben begleitet war. Die Vermutung liegt nach, dass der damit verbundene Verfall der demokratischen Responsivität staatlicher Politik durch die Maßnahmen der Staaten zur Bekämpfung der von den Banken verursachten Wirtschaftskrise um Jahre, wenn nicht Jahrzehnte beschleunigt worden ist.

\section{Ein langer Trend}

Die zusätzliche Verschuldung des deutschen Staates in Reaktion auf die „Second Great Contraction“" (vgl. Reinhart u. Rogoff 2009b), so immens und präzedenzlos sie auch sein mag, setzt eine Entwicklung fort, die Anfang der 1970er Jahre begann und nie gebrochen werden konnte. ${ }^{1}$ Egal unter welcher Regierung, in wirtschaftlich guten wie in schlechten Zeiten, vor der Wiedervereinigung und danach, die Staatsverschuldung ist immer nur gewachsen (Abb. 1). Die ganz wenigen Ausnahmen gingen auf ein niedriges Zinsniveau oder ein hohes Wirtschaftswachstum zurück, niemals aber auf Einnahmeüberschüsse in den öffentlichen Haushalten (für den Bund s. Abb. 2). ${ }^{2}$ Als Folge lagen die Ausgaben des Bundes für den Schuldendienst im Jahr 2005, am Ende der zweiten Regierung Schröder, bei etwa 14\% des Bundeshaushalts (Abb. 3); Ende der 1990er Jahre hatten sie schon einmal mehr als $16 \%$ ausgemacht und waren dann vor allem wegen günstiger Refinanzierungsmöglichkeiten auf den Weltkapitalmärkten ${ }^{3}$ zurückgegangen. Dennoch entsprachen die Zinszahlungen des Bundes im Jahre 2005 in ihrer Höhe etwa dem Haushaltsdefizit - was bedeutet, dass sämtliche neu aufgenommenen Schulden zur Begleichung der Zinsen auf die Altschulden verwendet werden mussten.

Ausmaß und Charakter der deutschen Staatsverschuldung bis zum Beginn der gegenwärtigen Krise erschließen sich durch einen Blick auf die bis Mitte des letzten Jahrzehnts entstandene Ausgabenstruktur. Zu diesem Zeitpunkt wandte der Bund mehr als ein Drittel seines Haushalts für Zuschüsse an die Rentenversicherung auf. Der rapide Anstieg dieses Ausgabenpostens ging auf die Entscheidung aller Parteien Mitte der 1990er Jahre zurück, den Gesamtsozialversicherungsbeitrag aus beschäftigungspolitischen Gründen nicht über vierzig Prozent steigen zu lassen; hieran haben sich sämtliche Regierungen seit 1996 mehr oder weniger gehalten. Ergebnis war, dass der Anteil der Zuschüsse zur Sozialversicherung am Bundeshaushalt innerhalb nur eines Jahrzehnts von einem Fünftel auf ein Drittel anstieg (Abb. 4). Zusammen mit den Zinsen auf die über Jahrzehnte hinweg aufgelaufenen Schulden gingen damit 2005 etwa die Hälfte der Bundesausgaben an Rentner und Rentiers. Hinzu kamen etwa $15 \%$ für die Grundsicherung von Arbeitslosen und Sozialhilfeempfängern (,Hartz IV“).

1 Zum Folgenden s. Streeck (2007).

2 Ohne die extensive Privatisierung von Staatseigentum seit Mitte der 1990er Jahre wären die jährlichen Defizite in der damaligen Zeit noch höher ausgefallen. Heute sind alle Privatisierungsmöglichkeiten weitgehend und irreversibel ausgeschöpft (vgl. Zohlnhöfer u. Obinger 2005).

3 Hierzu unten mehr. 


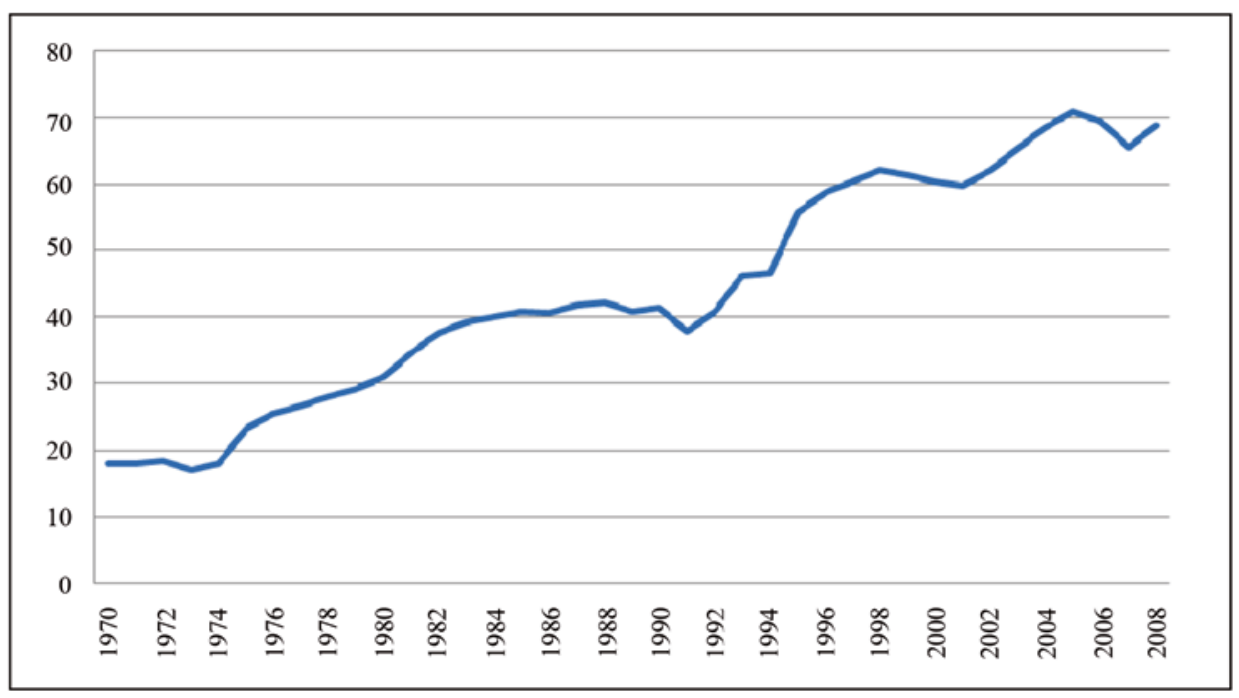

Abb. 1: Gesamtverschuldung der öffentlichen Haushalte, in Prozent des BIP, 1970-2008

Nimmt man alle Ausgabenposten zusammen, die sich kurz- und mittelfristig der politischen Disposition entziehen, ${ }^{4}$ so lag der politisch disponible Anteil am Bundeshaushalt 2008 bei $21,5 \%$; 1970 hatte er noch knapp $40 \%$ ausgemacht (Tab. 1). So dramatisch diese Zahlen auch erscheinen mögen, das wahre Ausmaß der Schrumpfung des politischen Entscheidungs- und Handlungsspielraums wird durch sie eher noch verborgen. Denn unterhalb der Nettogrößen konnte der Zuwachs des Anteils der Zuschüsse zur Sozialversicherung (von 13,5\% 1970 auf 34,3 \% 2009), der Grundsicherung für Arbeitsuchende (von unter einem auf 12,7\%) und des Schuldendienstes (von 3,1 auf 14,2\%) zwei „Friedensdividenden“ aufzehren: den Rückgang der Ausgaben für Kriegsfolgelasten aus dem Zweiten Weltkrieg und die Einsparungen im Verteidigungsetat nach 1989 (Abb. 5). Reserven in vergleichbarer Größenordnung - von nicht weniger als einem Fünftel der Gesamtausgaben (die Aufwendungen für vergangene und künftige Kriege beliefen sich 1970 auf $32,7 \%$ und 2009 nur noch auf 11,2\%) - wird es aber in Zukunft nicht mehr geben, so sehr sie auch gerade jetzt zum Ausgleich der anstehenden Belastungen durch die Neuverschuldung nach der Finanzkrise gebraucht würden. ${ }^{5}$

$4 \mathrm{Zu}$ ihnen gehören neben der sozialen Sicherung und dem Schuldendienst die Ausgaben für Verteidigung (die politisch nicht im Ermessen der Bundesregierung stehen, sondern sich aus ihren Bündnisverpflichtungen ergeben) sowie für Personal.

5 Dasselbe gilt für die Mobilisierung zusätzlicher Einnahmen durch Privatisierung von Staatseigentum. Hier sind für die Zukunft allenfalls noch die Deutsche Bahn und die Autobahnen übriggeblieben. 


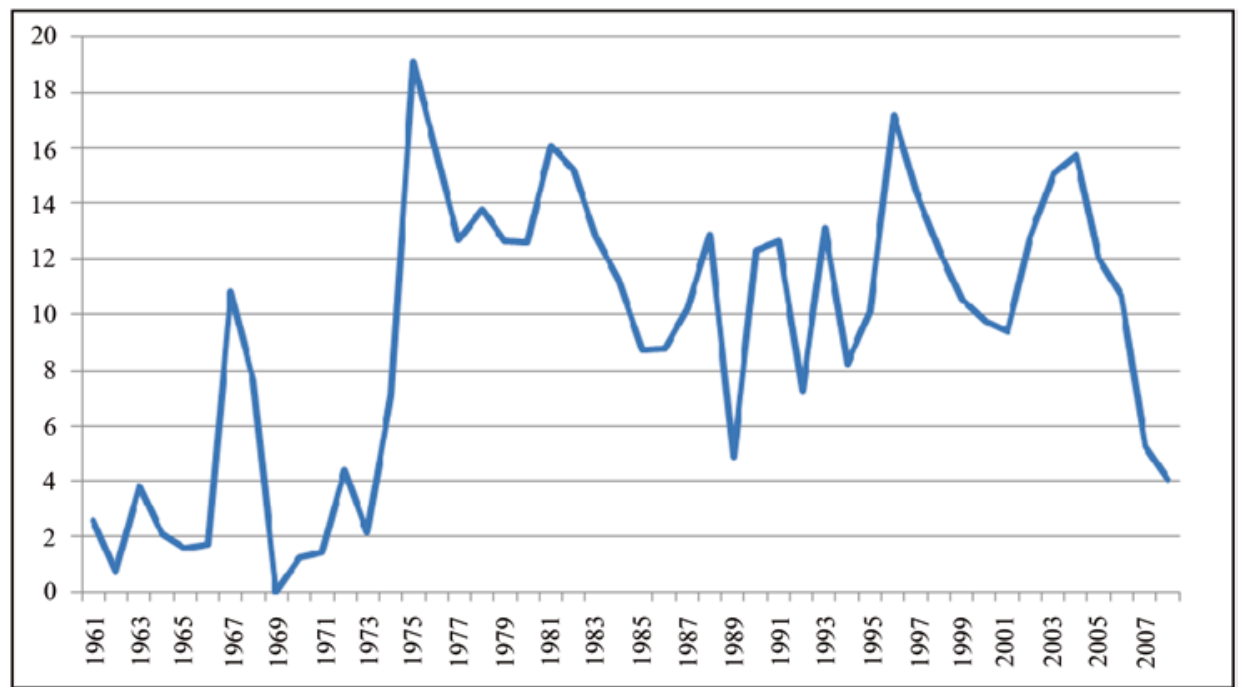

Abb. 2: Nettokreditaufnahme des Bundes, in Prozent der Bundesausgaben, 1961-2008

\section{Austerität als Regime}

Politisches Resultat des endemischen Defizits und der stetig wachsenden Verschuldung war ein allmählicher, aber nichtsdestoweniger schmerzhafter Übergang in ein Regime fiskalischer Austerität, das mit der letzten Regierung Kohl begann, unter der Kanzlerschaft Schröders erbitterte gesellschaftliche Auseinandersetzungen auslöste und von der Großen

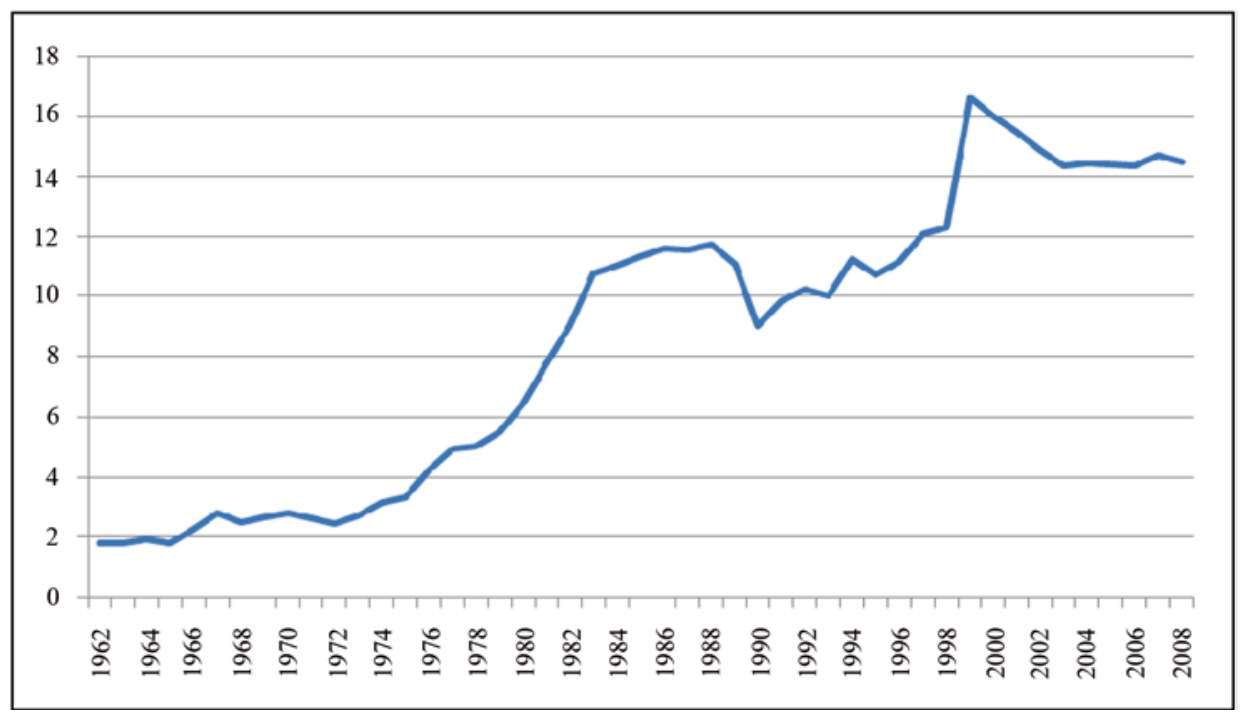

Abb. 3: Schuldendienst des Bundes, in Prozent der Bundesausgaben, 1962-2008 


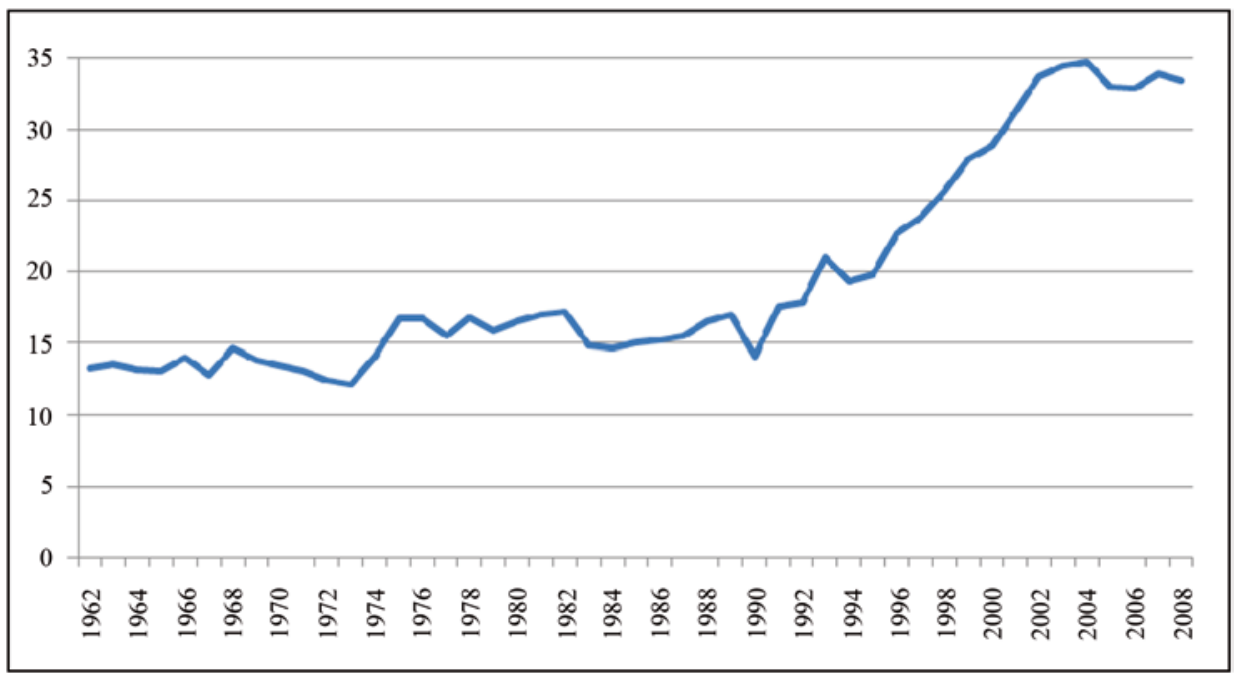

Abb. 4: Zuschüsse des Bundes zu den Sozialversicherungssystemen, in Prozent der Bundesausgaben, 1962-2008

Koalition dann - zunächst - gewissermaßen offiziell etabliert wurde. Ein ähnlicher Prozess hatte etwa ein Jahrzehnt vorher in den Vereinigten Staaten stattgefunden, wo er von Paul Pierson beschrieben worden war (vgl. Pierson 1998, 2001). Charakteristisch für das neue Regime, das die Nachkriegsperiode ,permissiver öffentlicher Finanzen“ (vgl. Steuerle 1996) ablöste, waren Pierson zufolge ein wachsender Widerstand gegen Steuererhöhungen und zunehmend populäre Forderungen nach Steuersenkungen; kontinuierliche, gewissermaßen eingebaute Ausgabensteigerungen bei den in der Nachkriegszeit

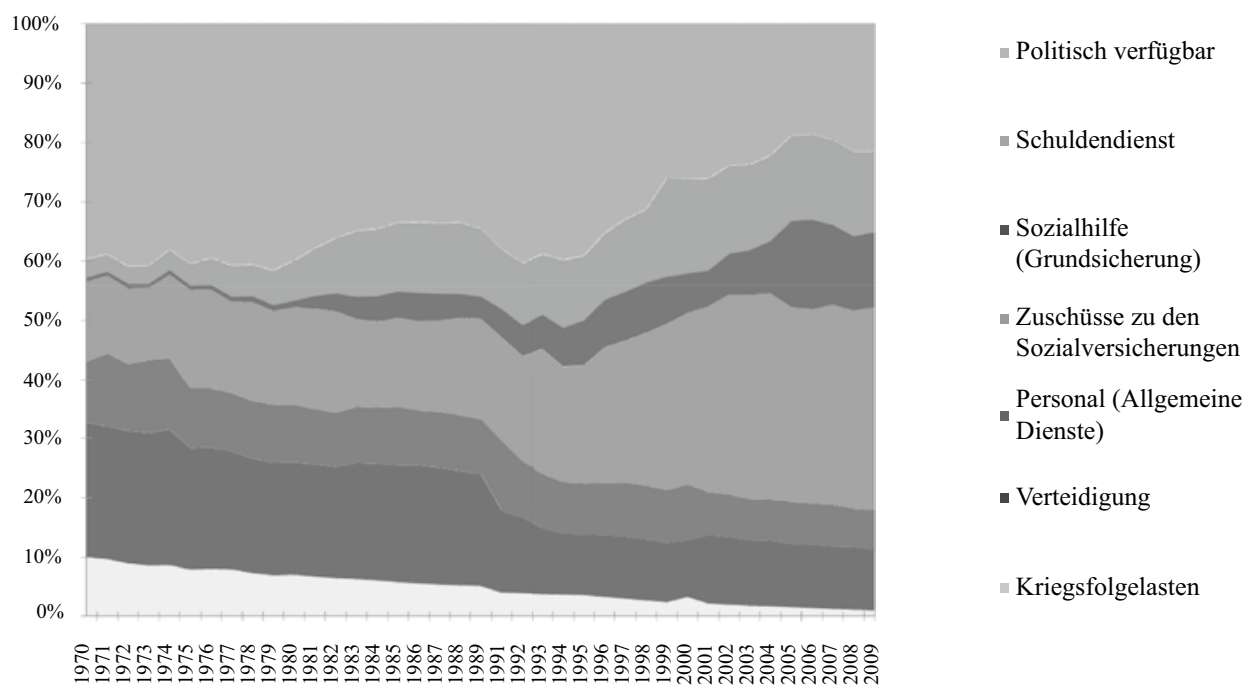

Abb. 5: Langfristig gebundene und politisch verfügbare Ausgaben im Bundeshaushalt, in Prozent, 1970-2009 
Tab. 1: Anteil der diskretionären Ausgaben am Bundeshaushalt, 1970 bis 2008

\begin{tabular}{ll}
\hline & Prozent \\
\hline 1970 & 39,6 \\
1975 & 40,4 \\
1980 & 39,9 \\
1985 & 33,4 \\
1989 & 34,5 \\
1995 & 39,1 \\
2000 & 26,0 \\
2005 & 18,8 \\
2006 & 18,6 \\
2007 & 19,5 \\
2008 & 21,5 \\
\hline
\end{tabular}

eingerichteten, nunmehr zur „Reife“ gelangenden Sozialversicherungssystemen (auch, aber nicht nur, aus demographischen Gründen); wachsende Staatsschulden als Folge hartnäckiger Defizite in den öffentlichen Haushalten, vergrößert durch stagnierendes Wirtschaftswachstum und das Ende der Inflation; immer neue, typischerweise marginale, aber sich über die Jahre addierende Kürzungen im politisch disponiblen Bereich der öffentlichen Haushalte, mit einer stetigen Zunahme des Anteils der feststehenden Ausgaben; und regelmäßige Versuche der jeweiligen Regierungen, den Zuwachs der Ausgaben für die ererbten Programme des Wohlfahrtsstaats einzudämmen - Versuche, die, wie die Beispiele Kohl 1998 und Schröder 2005 zeigen, durchaus mit dem politischen Ableben ihrer Betreiber enden können.

Hauptmerkmal, so Pierson, des neuen Austeritätsregimes ist die in ihm institutionalisierte „Fiskalisierung“ der Politik: die Bewertung jeder politischen Entscheidung unter dem Gesichtspunkt ihrer Auswirkung auf „das Defizit“ und die Beförderung der Konsolidierung der staatlichen Haushalte zum zentralen Thema des öffentlichen Diskurses und obersten Gebot des politischen Handelns. In Deutschland war dieser Zustand spätestens mit dem Amtsantritt der Großen Koalition im Jahr 2005 erreicht. Wie Pierson für die Vereinigten Staaten herausgearbeitet hat, steht Haushaltskonsolidierung als Staatsziel vor allem einer aktivistisch gestaltenden, in gesellschaftliche Verhältnisse intervenierenden Politik im Weg, weil jede neue Initiative weitere tiefe Eingriffe in alte Besitzstände erforderlich machen würde. Ergebnis ist ein Rückgang der demokratischen Responsivität und politischen Handlungsfähigkeit des Staates. Allerdings ist Konsolidierung, je weiter die Verschuldung fortschreitet, auch und zugleich Voraussetzung für deren Bewahrung und Wiederherstellung. In diesem Sinne wird sie von sozialdemokratischen Mitte-LinksRegierungen betrieben, die freilich - wie in den USA die Clinton-Administration - vor der Schwierigkeit stehen, ihren Anhängern und der Wählerschaft insgesamt Ausgabenkürzungen als Mittel zum Zweck zukünftiger Möglichkeiten zur Ausgabensteigerung und einer späteren Wiederbelebung interventionistischer Politik plausibel machen zu müssen. Liberalen und konservativen Parteien dagegen fällt Austerität leichter: für sie ist Konsolidierung nicht ein Umweg zu einem erneuert interventionistischen, sondern der direkte Weg zu einem „schlanken“ Staat (vgl. Pierson 2001). 


\section{Fiskalkrise und Finanzkrise}

Ein bislang kaum diskutiertes Thema sind die komplexen Zusammenhänge zwischen der chronischen Fiskalkrise des Staates und der gegenwärtigen globalen Krise der Finanzwirtschaft. Betrachtet man die Entwicklung des Bundeshaushalts im letzten Jahrzehnt genauer, so zeigt sich ein nicht unerheblicher Rückgang der Aufwendungen für den Schuldendienst seit dem Ende der 1990er Jahre (Abb. 3), und zwar trotz eines Anstiegs der Gesamtverschuldung des deutschen Staates um rund zehn Prozentpunkte gemessen am Bruttoinlandsprodukt (Abb. 1). Hauptursache hierfür ist eine längerfristige Verbesserung der Verschuldungsbedingungen in Gestalt eines Rückgangs der vom Staat auf seine Schulden zu zahlenden Zinsen. Im deutschen Fall sank der Zinssatz für langfristige Staatsanleihen nahezu kontinuierlich von 8,5\% 1991 auf 4,0\% 2008. Eine ähnlich starke Abnahme findet sich in allen vergleichbaren Ländern, beginnend in der ersten Hälfte der 1990er Jahre und hinführend auf eine bemerkenswerte Konvergenz um das Jahr 2000 (Abb. 6). Wie in Deutschland, so fällt auch in den meisten Vergleichsländern der Rückgang der Zinssätze nicht etwa mit einem Rückgang, sondern im Gegenteil mit einer Zunahme der Verschuldung zusammen, am dramatischsten in Japan, wo die Staatsverschuldung zwischen 1990 und 2008 von 65 auf $172 \%$ des Bruttoinlandsprodukts stieg.

Bei den aufgrund der niedrigen Zinsen eingesparten Haushaltsmitteln handelt es sich um erhebliche Summen. Im Jahr 2008 entsprach ein Prozentpunkt bei den durchschnittlich für die Staatschuld geltenden Zinssätzen einem Betrag von rund 3,5\% des deutschen Bundeshaushalts. Hätte der durchschnittliche Zinssatz bei sechs statt, wie hier der Einfachheit halber unterstellt, bei vier Prozent gelegen, ${ }^{6}$ so hätte ceteris paribus der politisch disponible Anteil der Bundesausgaben statt rund 21 nur noch 14\% ausgemacht. Zweifellos hätte dies die gesellschaftlichen Konflikte der damaligen Zeit noch weiter verschärft.

Nicht zu übersehen ist, dass die längerfristige Verbesserung der Refinanzierungsbedingungen der Staaten mit der Deregulierung der Finanzmärkte und der „Politik des leichten Geldes“ der amerikanischen Regierung und der Federal Reserve seit Anfang der 1990er Jahre zusammenfällt. ${ }^{7}$ Dies legt die Vermutung nah, dass es nicht nur die Banken waren, die von der von den Staaten ausgestellten Lizenz zu fast unbegrenzter privatwirtschaftlicher Liquiditätsproduktion profitiert haben, sondern auch die Staaten selber. Die einzigartig günstigen Refinanzierungsbedingungen in den 1990er und 2000er Jahren jedenfalls haben es den Staaten ermöglicht, sich weit stärker zu verschulden, als sie dies unter normalen Umständen gekonnt hätten. Hierin ähneln sie den Käufern von subprime mortgages, auch und vor allem, wenn man die nun notwendig gewordenen enormen Ausgaben zur vorläufigen Bewältigung der Finanzkrise als nachgelieferte Rechnung für die, wie sich herausstellt, nur scheinbar billigen Kredite der letzten Jahre ansieht. Greenspans „easy money“ (billiges Notenbankgeld) trat an die Stelle von „easy finance“ (wie von

6 Die durchschnittliche Verzinsung der Staatsschulden reagiert nur langsam und in dem Maße auf Veränderungen des Zinssatzes, wie neue Schulden aufgenommen und alte Schulden umfinanziert werden müssen.

7 Ein Jahrzehnt früher war es die Austilgung der Inflation, die zu einem Einbruch der Zinssätze führte; danach stiegen die Zinsen bis zum Beginn des folgenden Jahrzehnts wieder leicht an (s. Abb. 6). 


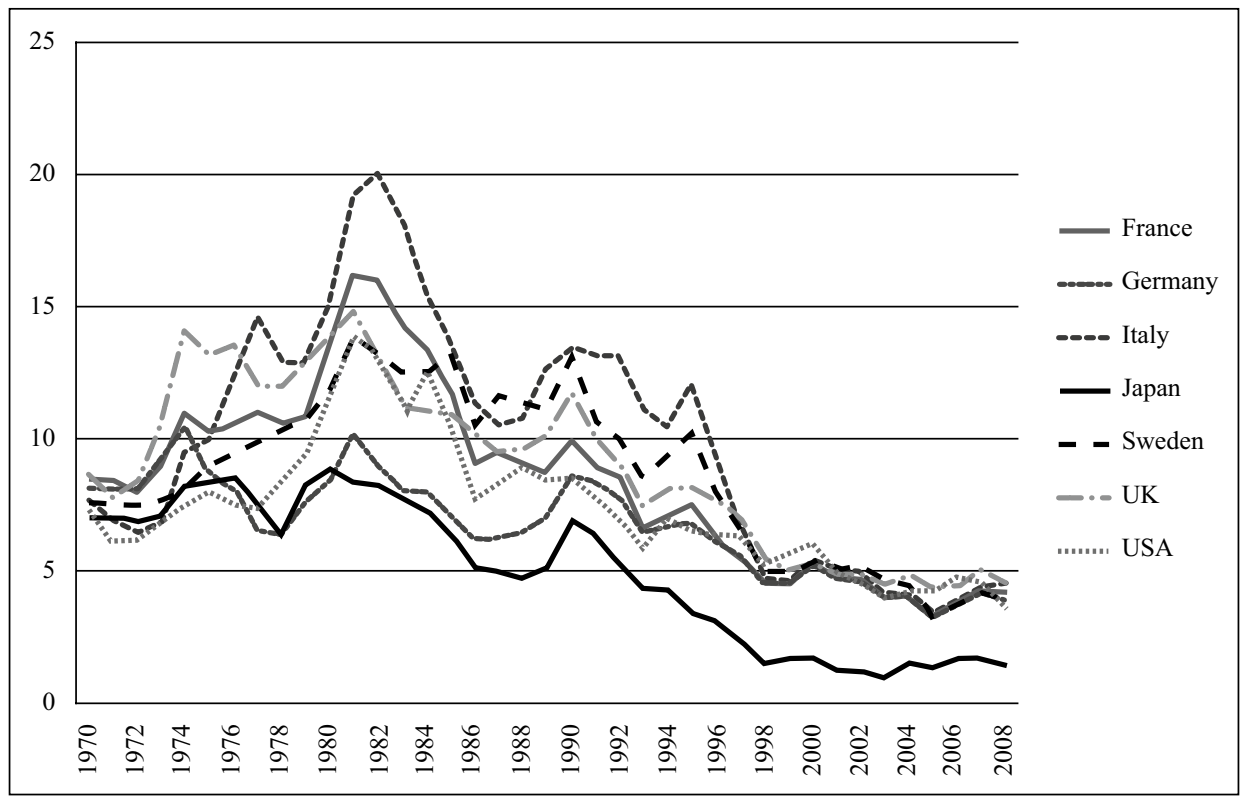

Abb. 6: Langfristige Verzinsung von Staatsanleihen

selbst wachsender Staatseinnahmen; vgl. Steuerle 1996) als staatliches Instrument zur Suspendierung gesellschaftlicher Konflikte und als temporärer Notbehelf zur Vermeidung von Einschnitten in den Wohlfahrtsstaat.

Was die Handlungsfähigkeit des Nationalstaats angeht, so wirft dies die Frage auf, ob die Staaten sich heute eine restriktive Re-Regulierung des Finanzsektors, wie sie für dessen nachhaltige Stabilisierung vermutlich erforderlich wäre, überhaupt leisten können, sofern diese, wie man eigentlich erwarten müsste, zu einer Geldverknappung und damit zu einem Anstieg des Zinsniveaus führen würde. Höhere Zinsen würden die Konsolidierung der Haushalte noch schwieriger und konfliktträchtiger machen, wobei sie ironischerweise zuerst auf die präzedenzlos hohe, zur Behebung der durch die Finanzkrise verursachten Flurschäden aufgenommene Neuverschuldung zu entrichten wären. Eine interessante sozialstrukturelle Folge wäre, dass die Mittelschichten sich immer mehr aus Steuerbürgern in Gläubiger des Staates verwandeln könnten, und mit erheblich verbesserter Marktposition. Auch wäre die Drohung mit höheren Zinsen ein wirksames Einschüchterungsmittel der Finanzwirtschaft gegenüber Regierungen, die den Versuch unternehmen wollten, den geld- und profitschöpferischen Handlungsspielraum der Banken durch verbesserte Regulierung einzuengen.

\section{Die Regierung Merkel/Westerwelle}

Als die derzeitige Bundesregierung Ende 2009 ihr Amt antrat, stand sie vor den Trümmern der Konsolidierungspolitik der Großen Koalition. Diese hatte in den Jahren 2005 
bis 2008 einen durchaus ehrgeizigen Versuch zur Sanierung der Staatsfinanzen unternommen. Die politischen und wirtschaftlichen Bedingungen hierfür waren günstiger gewesen denn je. Anders als Kohl oder Schröder verfügte die Große Koalition in Bundestag und Bundesrat über breite Mehrheiten, und die konjunkturelle Entwicklung 2006 und 2007 übertraf selbst optimistische Erwartungen. So ging die Arbeitslosigkeit von 2005 auf 2008 um 1,4 Mio. zurück, was zu einer erheblichen Entlastung der sozialen Sicherungssysteme führte. ${ }^{8}$ Die Große Koalition erhöhte die Mehrwertsteuer um drei Prozentpunkte, kürzte Steuervergünstigungen, fror das Rentenniveau zeitweilig ein und setzte das Rentenalter herauf. Wie schwierig die Konsolidierung allerdings schon damals war, lässt sich daran erkennen, dass der Finanzbericht der Bundesregierung vom Frühjahr 2008 trotz allem erst für 2011 mit einem ausgeglichenen Haushalt rechnete; von Schuldenabbau war allenfalls als Nebenfolge des damaligen, unbekümmert in die Zukunft prognostizierten Wirtschaftswachstums ${ }^{9}$ und unter der stillschweigenden Voraussetzung kontinuierlich niedriger Arbeitslosigkeit die Rede. Immerhin gelang es bis 2008, den fiskalischen Handlungsspielraum im Bundeshaushalt gegenüber 2005 um 2,7 Prozentpunkte zu erweitern.

Schon vor dem Zusammenbruch des Weltfinanzsystems im Herbst 2008 allerdings hatte der nahende Wahlkampf den Konsolidierungskonsens der Regierungsparteien auszuhöhlen begonnen. Die vor allem von Frankreich und Großbritannien geforderten Konjunkturprogramme, zunächst von der Bundesregierung nur widerwillig um des EU-Friedens willen beschlossen, wurden sehr bald als Gelegenheit genutzt, sich von der zunehmend ungeliebten Austeritätspolitik der vorangegangenen drei Jahre zu verabschieden und sich für die Bundestagswahl in Position zu bringen. Krisenbewältigung wurde zur Lizenz für Wähler- und Klientelbeglückung; siehe als besonders bizarre Beispiele den Wunsch der CSU, zur Bekämpfung der Finanzkrise den Milchbauern die Steuern auf Agrardiesel zu erlassen, sowie die hartnäckige Forderung - die Gründe kennt man nun besser - von FDP und CSU, zur Steigerung des Wirtschaftswachstums die Mehrwertsteuer auf Hotelübernachtungen um 12 Prozentpunkte zu senken.

Im Frühjahr 2009 waren dann alle Dämme gebrochen und man beschloss einhellig die Verlängerung der „Abwrackpauschale“ bis nach der Wahl. Niemand, auch die Opposition nicht, sprach mehr von „Sparen“; jede klientelistische Forderung konnte mit Verweis auf die Notwendigkeiten der Krisenbekämpfung oder auch auf die Riesensummen begründet werden, die für die Banken und Industrieunternehmen bereitgestellt worden waren. Hatte die CDU im Wahlkampf 2005 noch Steuererhöhungen angekündigt - und damit nach späterer Erkenntnis die Wahl beinahe verloren - so versprach sie diesmal im Gegenteil und in Einklang mit ihrem Wunschpartner FDP Steuersenkungen. Gleichzeitig wurde zur diskurspolitischen Entsorgung der Konsolidierungsrhetorik der Jahre nach 2005 zusammen mit der SPD eine monumentale Verfassungsänderung verabschiedet, mit der der Staat sich für die mittelfristige Zukunft zu ausgeglichenen Haushalten und zum Abbau seiner Altschulden verpflichtete. Keiner der Erfinder der sogenannten „Schuldenbremse“

8 Beispielsweise konnte der Beitragssatz zur Arbeitslosenversicherung von 2005 bis 2009 um nicht weniger als 3,7 Prozentpunkte gesenkt werden, was trotz der Sparpolitik fällig werdende Beitragserhöhungen in anderen Bereichen mehr als ausglich.

92006 wuchs die deutsche Volkswirtschaft um 3,0\%, 2007 immer noch um 2,5\%. In den zehn Jahren davor hatte das durchschnittliche Wachstum pro Jahr bei 1,3\% gelegen. 
hat jemals erklärt, wie diese Verpflichtung eingehalten werden soll. Vermutlich ging es bei ihr wirklich allein darum, der Politik den Rücken für den Aufbau neuer Schuldenberge freizumachen.

Nach ihrem Wahlsieg im September 2009 müssen die bürgerlichen Parteien Wege finden, mit dem widersprüchlichen Erbe einer präzedenzlosen Neuverschuldung, eines nunmehr mit Verfassungsrang ausgestatteten Konsolidierungsprogramms und ihrer Wahlversprechen zurechtzukommen. Was die Konsolidierung der öffentlichen Haushalte angeht, so haben die zur Krisenbekämpfung neu aufgenommenen Schulden sämtliche Sanierungserfolge der Großen Koalition zunichte gemacht. Defizit und Schuldenlast werden in den kommenden Jahren unvergleichlich größer sein als zum Ende der zweiten Regierung Schröder. ${ }^{10}$ Alles, was die Große Koalition unter Aufwendung ihrer beträchtlichen politischen Kräfte erreichen konnte, verblasst vor dem, was heute an Einschnitten nötig wäre, wenn dem neuen Konsolidierungsgebot des Grundgesetzes Genüge getan werden soll. Dabei ist die politische Basis der zweiten Regierung Merkel nicht annähernd so breit wie die der ersten. Freilich, eine Rückkehr zur Politik der Großen Koalition hatten die neuen Regierungsparteien ohnehin schon im Wahlkampf ausgeschlossen und sie, entgegen manchen Erwartungen, auch nach der Wahl nicht einmal versucht. Der Koalitionsvertrag für die Jahre 2009 bis 2013 schließt denn auch wie versprochen nicht nur Steuererhöhungen aus, sondern kündigt sogar neue Steuersenkungen in jährlicher Gesamthöhe von 24 Mrd. Euro an, die „möglichst zum 1.1. 2011 in Kraft treten“ sollen, zusätzlich zu den schon im Wahljahr gemeinsam mit der SPD beschlossenen und Anfang 2010 wirksam werdenden Steuersenkungen von jährlich 14 Mrd. Euro (Christlich-Demokratische Union et al. 2009, S. 10 f.).

Die Steuersenkungspläne der zweiten Regierung Merkel sind in der Öffentlichkeit wegen ihrer Unvereinbarkeit mit den fiskalpolitischen Vorgaben der Verfassung scharf kritisiert worden. So schließt der Bundesrechnungshof aus seiner Analyse der Schuldenentwicklung des Bundes in scharfem Widerspruch zur Bundesregierung, dass „derzeit keine finanziellen Spielräume für schuldenfinanzierte Steuersenkungen oder Ausgabensteigerungen“ bestünden (Bundesrechnungshof 2009, S. 74, ebenso S. 83). Insbesondere verweist er auf die absehbar steigenden Zinslasten, die 2014 selbst bei konstanten Zinssätzen um 11 Mrd. Euro höher sein würden als 2009 (Bundesrechnungshof 2009, S. 83). Darüber hinaus seien bei einem jederzeit möglichen „Anstieg des gegenwärtig niedrigen Zinsniveaus erhebliche zusätzliche Belastungen“ zu erwarten (ibid., S. 74).

Ähnlich erinnert der Sachverständigenrat zur Begutachtung der gesamtwirtschaftlichen Entwicklung (SVR) in seinem Jahresgutachten 2009/10 daran, dass zur Reduzierung der Neuverschuldung des Bundes auf 0,35\% des Bruttoinlandsprodukts im Jahr 2016, wie

102005 betrug der Finanzierungssaldo des Bundeshaushalts 31,4 Mrd. Euro (12,1\% der Bundesausgaben). Für 2009 rechnet der Bundesrechnungshof mit einem Defizit von 49,5 Mrd. (16,3\%) und für 2010 mit einem weiteren Anstieg auf 86,4 Mrd. (26,4\%) (Bundesrechnungshof 2009, S. 76). Die Gesamtverschuldung des Bundes 2005 machte $40 \%$ des Sozialprodukts aus; 2010 wird sie bei 45 und 2013, allerdings nur bei Einhaltung der neuen Schuldenregel (!), bei $49 \%$ liegen (Bundesrechnungshof 2009, S. 107). Für den Schuldenstand des Gesamtstaats rechnet der Sachverständigenrat mit einen Anstieg von 67,6\% im Jahr 2005 auf 79\% 2010 und 82\% 2013 (Sachverständigenrat zur Begutachtung der gesamtwirtschaftlichen Entwicklung 2009, S. 184). 
von der Verfassung nunmehr zwingend vorgeschrieben, eine Haushaltslücke von $37 \mathrm{Mrd}$. Euro geschlossen werden müsste (2009, S. 179 ff.). Wenn mit der Konsolidierung 2011 begonnen würde, könnten die erforderlichen Maßnahmen über sechs Jahre verteilt werden und würden dann jährlich 6 bis $7 \mathrm{Mrd}$. Euro ausmachen - allerdings nur unter der Voraussetzung des von der Bundesregierung angenommenen, vom Sachverständigenrat zurückhaltend als ,optimistisch“ bezeichneten durchschnittlichen jährlichen Wirtschaftswachstums von nominal immerhin $3 \frac{1}{4} \%$ (ibid., S. 182, 185). ${ }^{11}$ Selbst dann aber erscheint eine Konsolidierung ohne Steuerhöhungen dem Sachverständigenrat unerreichbar, weil nicht damit zu rechnen sei, ,dass die Politik ... die Kraft für [entsprechend; WS] weitreichende (und bislang nie dagewesene) Ausgabenkürzungen“" aufbringen könne. Da „dauerhaft schuldenfinanzierte Steuersenkungen ... finanzpolitisch unseriös und mit den grundgesetzlichen Regelungen zur Schuldenbegrenzung nicht vereinbar" seien, rät der SVR der Regierung ausdrücklich, doch noch über Steuererhöhungen nachzudenken (ibid., S. 189).

Dies aber könnte vor allem die FDP wohl nur bei Strafe des politischen Untergangs. Auch sonst sind der Regierung die Hände weitgehend gebunden. Was die von ihr verbreiteten Hoffnungen auf eine „Konsolidierung durch Wachstum und Steuersenkungen angeht, so bezeichnet der SVR diese in einer Zwischenüberschrift respektlos als Tagträumereien“ (ibid., S. 185). Da ein Schuldenabbau durch Inflation unter den derzeitigen Bedingungen nur gegen den Widerstand der Europäischen Zentralbank durchsetzbar wäre, blieben für eine „bürgerliche“ Regierung als Mittel der Wahl nur Kürzungen bei den Sozialausgaben. Hier aber ist die Erinnerung an die traumatischen Auseinandersetzungen um die Hartz IV-Reformen noch allzu lebendig. Die Maßnahmen der Schröderschen „Agenda 2010“ haben ohnehin, zusammen mit den vorher unter Kohl und nachher unter der Großen Koalition vorgenommenen weiteren Einschnitten, den Spielraum für zusätzliche Einsparungen nachhaltig verkleinert; der größte Teil dessen, was heute als Konsolidierungsreserve dienen könnte, ist bereits weggespart. Beispielsweise liegt die Eckrente schon heute gefährlich nah am Sozialhilfesatz und damit an einem Punkt, wo die Verfassungsmäßigkeit des beitragsfinanzierten Rentensystems zum Problem werden könnte. Weitere Einschnitte in soziale Leistungen sind deshalb nicht ausgeschlossen und sind im Gegenteil sogar zu erwarten; sie werden aber nicht dramatisch ausfallen können und sich über die Jahre verteilen müssen. ${ }^{12}$

Was die Aussichten auf greifbare Konsolidierungserfolge angeht, so hat es Reinhart und Rogoff zufolge bei ähnlichen Finanzkrisen in der Zeit nach dem Zweiten Weltkrieg im Durchschnitt 4,4 Jahre gedauert, bis die Wirtschaft wieder auf das Niveau vor der Krise

11 In den zehn Jahren von 1999 bis 2008 lag das durchschnittliche nominale Wirtschaftswachstum bei nur 2,4\%. Laut Sachverständigenrat würde ein Wachstum auf Vorkrisenniveau ,den Konsolidierungsbedarf um etwa ein Drittel höher ausfallen lassen“ (Sachverständigenrat zur Begutachtung der gesamtwirtschaftlichen Entwicklung 2009, S. 186). Gegen Ende des Jahres 2009 rechnete der Finanzminister schon mit einem jährlichen Konsolidierungsbedarf von $10 \mathrm{Mrd}$. Euro ab 2011 (FAZnet, 21.12.2009) - was darauf hindeutet, dass die Bundesregierung dabei ist, ihre Wachstumserwartungen nach unten zu revidieren.

12 Allerdings können sie sich durchaus addieren. Auch durch allmähliches „,bleeding from a thousand cuts" kann der Wohlfahrtsstaat heruntergefahren werden. 
gewachsen war (Reinhart u. Rogoff 2009b, S. 236). ${ }^{13}$ Wegen des Ausmaßes der Krise von 2008 könnte die Erholung diesmal noch länger brauchen. Darüber hinaus scheint es Anzeichen zu geben, dass Staatschulden jenseits einer gewissen Größenordnung das Wachstum zusätzlich verlangsamen; der Schwellenwert scheint irgendwo in der Nähe von $90 \%$ des Sozialprodukts zu liegen (vgl. Reinhart u. Rogoff 2009a). ${ }^{14}$ Niedriges Wachstum bedeutet höhere Arbeitslosigkeit; diese wiederum bedeutet niedrigere Steuer- und Beitragseinnahmen, die die Konsolidierung der Haushalte weiter erschweren. Damit aber verschiebt sich der Punkt, an dem eine Regierung aus einer von ihr betriebenen, erfolgreichen Sanierung der öffentlichen Finanzen Nutzen ziehen könnte, mit hoher Gewissheit in die Zeit nach dem Ende ihrer Amtsperiode. Die Frage ist: würde eine CDU/CSU/FDP-Regierung unter absehbar beträchtlichen politischen Kosten die öffentlichen Haushalte konsolidieren, um dadurch die interventionistischen und redistributiven Handlungsmöglichkeiten einer Nachfolgeregierung aus SPD, Grünen und Linkspartei zu erweitern?

So wird es wohl beim Dauerdefizit bleiben, und die politische Kreativität der neuen Bundesregierung wird sich darauf konzentrieren, durch geschickte Buchführung Wege zu finden, um die Verschuldungsgrenzen des Grundgesetzes und des Maastrichter Vertrages zu umgehen. Das heißt allerdings nicht, dass die Regierung Merkel/Westerwelle aus dem schon vor der Neuverschuldung etablierten Austeritätsregime ausbrechen wird. Defizit und Schulden werden bleiben, aber eben deshalb auch weiterhin dazu dienen können, jeden Versuch einer aktivistisch-gestaltenden Politik links von der Mitte zu delegitimieren. Gerade weil die Handlungsfähigkeit der Regierung nach der Krise für eine Konsolidierung nicht ausreicht, reicht sie schon gar nicht für eine Überwindung des Austeritätszwangs. Weiter wachsende Schulden müssen aus der Sicht bürgerlicher Parteien kein zu hoher Preis für dessen Verewigung sein, solange Steuererhöhungen ausgeschlossen und Steuersenkungen Bestandteil der herrschenden politischen Formel und damit des klientelpolitischen Instrumentenkastens bleiben. ${ }^{15}$ Wie wir aus der vergleichenden Forschung wissen, können bürgerliche Regierungen ohnehin besser mit Defiziten und hohen

13 Die Rechnung bezieht sich auf 14 größere Finanzkrisen in 14 Ländern.

14 „Seldom do countries simply ,grow“ their way out of deep debt burdens“ (Reinhart u. Rogoff 2009a, S. 23).

15 Aus Sicht derer, die mit ihrem Geld lieber Staatsanleihen kaufen als es beim Finanzamt abzuliefern, empfiehlt sich ohnehin eine Politik des steuerpolitischen Ausverkaufs, solange der Vorrat reicht. Dasselbe gilt für die den Ausverkauf betreibenden Parteien, sofern es ihnen gelingt, sich ihre Gefälligkeiten, bevor endgültig keine mehr möglich sind, durch Spenden vergüten zu lassen. Möglicherweise kann man die Steuersenkungspläne der bürgerlichen Koalition aber auch als weltklugen Verzicht auf den Versuch betrachten, etwas zu erreichen, was man als unerreichbar erkannt hat. Oder es steckt, mindestens bei der Teilen der FDP, eine Strategie der Aushungerung und Abmagerung des Staates dahinter, wie sie in den 1980er Jahren sowie später unter Bush II in den USA unter dem Slogan „starving the beast“ erfolgreich betrieben wurde: Steuersenkungen gerade im Defizit, mit dem Ziel, die Staatsausgaben und damit die staatliche Handlungsfähigkeit langfristig zu vermindern. George W. Bush, 2001: „So we have the tax relief plan [...] that now provides a new kind - a fiscal straightjacket for Congress. And that's good for the taxpayers, and it's incredibly positive news if you're worried about a federal government that has been growing at a dramatic pace over the past eight years and it has been." Siehe http://en.wikipedia.org/wiki/Starve_the_beast, abgerufen am 21. Januar 2010. 
Staatsschulden leben als sozialdemokratische (vgl. Wagschal 1996): Defizite liefern gute Argumente gegen gestaltende oder umverteilende Politik, während Steuererhöhungen zu Lasten der eigenen Klientel gehen würden, die Zinsen auf eine wachsende Staatsschuld dagegen sichere Einkommen für die Käufer der Staatsanleihen in den begüterteren Schichten bedeuten.

\section{Politik im Defizit}

Damit wird im Gefolge der Finanzkrise die Sorge um die Staatsfinanzen und das Defizit mehr denn je und für alle praktischen Zwecke endgültig zum Zentralthema staatlicher Politik und sich wie Mehltau auf alle Diskussionen über mögliche neue staatliche Auf- und Ausgaben legen. Eine fiskalische Notmaßnahme wird der anderen folgen; Haushaltssperren, globale Minderausgaben und Nachtragshaushalte werden noch mehr als im abgelaufenen Jahrzehnt den Alltag der Politik bestimmen; und immer neue Konsolidierungsversuche werden immer wieder hinter ihren Zielen zurückbleiben. Aber für das Funktionieren des Austeritätsregimes ist ja, wie gesagt, die Bekämpfung des Defizits ohnehin wichtiger als seine Beseitigung; gerade hier gilt, dass der Weg das Ziel ist. Beharrungskraft und Wachstum der ererbten Pflichtausgaben und regelmäßige Einschnitte in die noch disponiblen Ausgaben werden den Anteil der letzteren am Staatshaushalt und damit die fiskalische Responsivität staatlichen Handelns kontinuierlich weiter verringern und Politik perspektivisch auf die Verwaltung von in der Vergangenheit begründeten privaten Ansprüchen an die öffentlichen Finanzen reduzieren. Dies könnte dazu führen, dass Demokratie für diejenigen, die aus einer gestaltenden Politik Nutzen ziehen würden, immer uninteressanter wird.

In der überschaubaren Zukunft dürfte nicht nur in Deutschland die Hauptbeschäftigung der nationalen Regierungen im Abstottern der durch die Krisenbekämpfung noch einmal in neue Dimensionen gewachsenen Staatsschulden bestehen; alles, was darüber hinausgeht, erscheint unter heutigen Bedingungen auf lange Zeit utopisch. Besonders entmutigend wird sein, dass die Tendenz hierzu weit vor die Krise zurückgeht und längst chronisch geworden zu sein scheint. Auch wird jede Regierung nach dem, was geschehen ist, wissen, dass eine neue Wirtschaftskrise - und wer könnte sie ausschließen? - alle Erfolge eines erneuten Anlaufs zur Wiederherstellung fiskalischer Handlungsspielräume wie gehabt auslöschen und die für sie erbrachten politischen Opfer ein weiteres Mal sinnlos machen würde. Dass die Regierung Merkel/Westerwelle unter diesen Umständen darauf verzichtet, ihr politisches Kapital für die Sisyphusarbeit eines neuen Sanierungsversuchs aufzubrauchen, der noch dazu weit schmerzhafter ausfallen müsste als der der Großen Koalition, erscheint so gesehen nicht unbedingt nur zynisch - auch wenn die Erwägung durchaus eine Rolle spielen dürfte, dass die Früchte einer erfolgreichen Haushaltskonsolidierung bestenfalls der Nachfolgeregierung zugutekommen würden. Könnte es sein, dass am Beginn des 21. Jahrhunderts die Daueraufgabe der Stabilisierung der kapitalistischen Marktwirtschaft die Kräfte des Nationalstaats endgültig erschöpft hat? 


\section{Literatur}

Bundesrechnungshof. 2009. Bemerkungen 2009 zur Haushalts- und Wirtschaftsführung des Bundes. Bonn: Bundesrechnungshof, Referat für Öffentlichkeitsarbeit.

Christlich-Demokratische Union, Christlich-Soziale Union, und Freie Demokratische Partei. 2009. Wachstum. Bildung. Zusammenhalt. Der Koalitionsvertrag zwischen CDU, CSU und FDP. Berlin.

Ferguson, Thomas, und Robert Johnson. 2009a. Too big to fail: The „Paulson Put“, presidential politics, and the global financial meltdown. Part I: From shadow financial system to shadow bailout. International Journal of Political Economy 38(1): 3-34.

Ferguson, Thomas, und Robert Johnson. 2009b. Too big to fail: The „Paulson Put“, presidential politics, and the global financial meltdown. International Journal of Political Economy 38(2): $5-45$.

Pierson, Paul. 1998. The deficit and the politics of domestic reform. In The social divide. Political parties and the future of activist government. Hrsg. Margaret Weir, 126-178. Washington DC and New York: Brookings Institution Press and Russell Sage Foundation.

Pierson, Paul. 2001. From expansion to austerity. The new politics of taxing and spending. In Seeking the center. Politics and policymaking at the new century. Hrsg. Martin A. Levin et al., 54-80. Washington DC: Georgetown University Press.

Reinhart, Carmen M., und Kenneth S. Rogoff. 2009a. Growth in a time of debt. Unpublished Manuscript.

Reinhart, Carmen M., und Kenneth S. Rogoff. 2009b. This time is different. Eight centuries of financial folly. Princeton: Princeton University Press.

Sachverständigenrat zur Begutachtung der gesamtwirtschaftlichen Entwicklung. 2009. Die Zukunft nicht aufs Spiel setzen. Jahresgutachten 2009/10. Wiesbaden: Statistisches Bundesamt.

Steuerle, C. Eugene. 1996. Financing the American state at the turn of the century. In Funding the modern American state, 1941-1995. The rise and fall of the era of easy finance. Hrsg. Elliott W. Brownlee, 409-444. Cambridge: Cambridge University Press.

Streeck, Wolfgang. 2007. Endgame? The fiscal crisis of the German state. MPIfG Discussion Paper 07/7. Köln: Max-Planck-Institut für Gesellschaftsforschung.

Wagschal, Uwe. 1996. Staatsverschuldung. Ursachen im internationalen Vergleich. Opladen: Leske \& Budrich.

Zohlnhöfer, Reimut, und Herbert Obinger. 2005. Ausverkauf des „Tafelsilbers“. Bestimmungsfaktoren der Privatisierungserlöse in EU- und OECD-Staaten 1990-2000. Politische Vierteljahresschrift 46(4): 602-628. 